\title{
Pituitary and hypothalamic physiology
}

\author{
G. M. BESSER
}

From the Department of Endocrinology, St Bartholomew's Hospital, London EC1A 7BE

Professor P. M. Daniel has described in the first paper the nervous pathways connecting the hypothalamus to the posterior pituitary and how the posterior pituitary merely stores the hormones vasopressin, oxytocin (which has no clearly defined function in man), and neurophysin, the large molecule or molecules which bind the other hormones. For many years the anterior pituitary was thought to be very different but recently it has been realised that conceptually it is quite similar.

\section{Anterior pituitary hormones}

The hormones produced by the anterior pituitary are growth hormone $(\mathrm{GH})$, prolactin (PRL), thyrotrophin (TSH), gonadotrophins (FSH and LH), and adrenocorticotrophin (ACTH). The existence of prolactin in the human was usually denied before 1970 , but since then it has been shown not only to be present but also to be of extreme importance in the control of lactation and gonadal function. In lower animals it also probably takes part in the control of the adrenal cortex and possibly in the regulation of salt and water metabolism. Disorders of prolactin secretion are remarkably common, especially in patients with infertility, and this has led to a reappraisal of pituitary function in reproduction. The melanocyte stimulating hormone (MSH) does not exist in man. The small peptide once known as MSH in man is an artefact and is derived from a much bigger hormone, lipotrophin, as a result of certain procedures carried out in vitro. Lipotrophin itself has very little pigmentary activity, and the only pigmentary hormone that circulates in man is ACTH. In Nelson's syndrome-that is, hyperpigmentation after adrenalectomy for Cushing's disease-and in Addison's disease the pigmentation is due to ACTH.

\section{REGULATORY HORMONES}

The secretion of the anterior pituitary hormones is controlled by other regulatory hormones called releasing or release-inhibiting hormones synthesised, like the posterior pituitary hormones, in the hypothalamus - as shown by the classical work of Harris (1955), now so clearly vindicated despite the extraordinary controversies of 20 years ago. Like the posterior pituitary hormones, the hypothalamic hormones are secreted by axonal flow, which takes $\frac{0}{8}$ them to the point where the pituitary stalk joins the is hypothalamus at the median eminence. There they $\vec{v}$ are stored as granules in swellings at the ends of the nerve fibres, closely applied to the portal capillaries $\infty$ which carry them into sinuses surrounding the $\frac{\circ}{3}$ anterior pituitary cells, where they can control the $\vec{c}$ synthesis and either stimulate or inhibit the release of the anterior pituitary hormones.

The hypothalamic hormones in fact form the first stage of a cascading amplifier. It has been cal- $\vec{\theta}$ culated that the human hypothalamus contains only about $1 \mathrm{ng}$ of any one releasing hormone, and the amount released in a short period into the portal capillaries is extremely small. However, the amount of trophic hormone released from the pituitary in response to the signal from the hypothalamus is about a million times as much. Further amplification occurs, probably of the same order, when the trophic hormone acts on the target gland. There is still further amplification when the secretion of the target glands acts on the body to produce metabolic effects which, in turn, produce behavioural changes in the individual.

The amplifier thus has three stages with a very precise sensing system for feedback control. The target gland secretions testosterone, oestrogens, thyroxine, tri-iodothyronine (T3), and cortisol can 은 act at the pituitary level to interfere with the influence of the hypothalamic hormone on the anterior pituitary cell-for example, T3 or TSH releasing cells. There is probably a similar negative feedback at the $O$ hypothalamic level, although this is somewhat $N$ controversial, so that the amount of hypothalamic N hormone released is also dependent upon sensing the $O$ target gland hormone level in the blood. There is, of

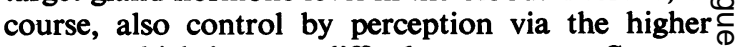
centres, which is more difficult to measure. Sensory $\stackrel{\infty}{+}$ stimuli such as vision, pain, fear, and so on, interact 0 with the regulatory mechanisms in the reticular $\overrightarrow{0}$ formation and the hypothalamus.

RELEASING HORMONES

Several releasing hormones are known to exist. They tend to be called factors when their structure is 
unknown. The first one to have its structure fully elucidated was the releasing hormone for thyrotrophin (TRH). There is also a releasing hormone for growth hormone, but its structure is not yet known. Two releasing hormones for the gonadotrophins, one for LH and one for FSH, were suggested by the original studies of Harris (1955) and McCann and Dhariwal (1964), but it is now thought that there is only one which causes the release of both gonadotrophins $\left(\mathrm{GnRH}^{*}\right)$. There is also a releasing factor of unknown structure for ACTH(CRF). Vasopressin acts in this way but the evidence suggests that it is not the physiological CRF. Prolactin release is also influenced by a releasing factor which, however, is not very important (see below).

There is good evidence that the releasing hormones cause the synthesis as well as the release of anterior pituitary hormones. Trophic hormones are stored within the anterior pituitary cell in two fractions, only one of which can be released rapidly. The first effect of all the releasing hormones is to release the readily available store, acting almost certainly through cyclic adenosine monophosphate (cyclic AMP). Subsequently they mobilise the non-releasable pool and then replenish it - that is, they produce synthesis as well as reorganisation of the compartments within the cell.

RELEASE-INHIBITING HORMONES

There are also release-inhibiting hormones. Growth hormone release-inhibiting hormone (GHR-IH) is otherwise known as somatostatin. This fascinating hormone does more than inhibit the release of growth hormone (see below). A prolactin releaseinhibiting hormone or factor (PIF) is the most important controlling factor for prolactin and will be discussed in more detail later. There is experimental evidence for a release-inhibiting factor for TSH, but TRH controls the secretion of TSH very effectively and the release-inhibiting hormone probably does not exist as a separate entity.

These hormones have been demonstrated by immunohistochemical methods. They seem to be discretely located in the median eminence although the cells which synthesise them are located at a distance from it. Thus the cells whose fibres carry neurophysin and vasopressin exist in the innermost layer of cells around the third ventricle, together with those producing GHR-IH, and the gonadotrophin releasing hormone seems to be in the outer layer.

* GnRH was originally called LHRH because that is its prime action. Since it also releases FSH it was later called LH/FSHRH. Gonadotrophin releasing hormone is a better name.

\section{Clinical importance of hypothalamic hormones}

Those most important clinically are thyrotrophin releasing hormone, gonadotrophin releasing hormone, growth hormone release-inhibiting hormone, and prolactin inhibiting factor, or prolactin releaseinhibiting hormone, which is almost certainly dopamine and not a peptide.

THYROTROPHIN RELEASING HORMONE

This is a tripeptide, pyro-glutamylhistidyl-prolineamide, with the pyro-glutamyl configuration which stabilises it and is necessary for its biological activity. It produces release of TSH very rapidly. Thus within three to four minutes of starting an intravenous infusion of TRH the plasma TSH begins to rise.

The specificity of the releasing hormones is of great interest. Thus on infusing TRH prolactin is secreted just as rapidly as TSH. Moreover, the effect on prolactin is more dramatic since it is secreted in a pulsatile fashion. There is reasonable evidence that TRH is not the physiological prolactin-releasing hormone, but in myxoedema high prolactin levels do occur, possibly because of excessive secretion of TRH.

An even more perplexing phenomenon is that in the male, but not in the female, TRH also causes some release of FSH. There is both in-vivo and in-vitro evidence that this is not an artefact due to reactivity between FSH and TSH in the assay. For instance, the FSH response can be suppressed by oestrogen, which, however, in the male, enhances the TSH response. Though of great interest, this effect is unimportant clinically.

The release of TSH by TRH is totally inhibited by thyroid hormones, hence the absence of a TSH response to TRH in thyrotoxicosis. But prolactin release is not suppressed to the same degree, though there is some impairment of the release of prolactin in thyrotoxic patients.

\section{GONADOTROPHIN RELEASING HORMONE}

This is a decapeptide (Schally et al., 1971), also with a pyro group on the front, having an amine at the end; it is available in synthetic form. A number of analogues with a longer action have been synthesised, and these have exciting potential for treating infertility. Other analogues have been made which bind to tissue receptors and, after brief stimulation, block them. This is shown by the absence of a response to gonadotrophin releasing hormone after giving the analogue. Currently the ratio of antagonism to agonism is not great enough for these analogues to be used as contraceptives but may become so with future developments.

As mentioned previously, it was thought that 
there were going to be two gonadotrophin releasing hormones. However, experiments on animals suggested that the decapeptide released both gonadotrophins, and in humans we found that when injected intravenously into a normal adult it released both LH and FSH, though the response of LH was much greater than that of FSH. Its effect lasts for about six hours, with a peak at between about 20 and 60 minutes. The effect can be abolished in women by giving large doses of oestrogens and in men by small doses of oestrogens. Surprisingly, it is not very sensitive to testosterone. Men have to be given high doses of testosterone for some time before the pituitary response to GnRH is abolished. Many, including ourselves, believe that testosterone may have to be converted to oestrogen before it will inhibit the pituitary response to GnRH.

In women the response to the gonadotrophin releasing hormone depends upon the stage of the menstrual cycle. This is partly due to variations in the responsiveness of the pituitary to GnRH throughout the menstrual cycle. Much more releasing hormone is secreted on the day of the $\mathrm{LH}$ peak than either before or after, and there is very good evidence that the gonadotrophin releasing hormone promotes synthesis as well as release of LH and FSH. Apparently during the menstrual cycle the pituitary content of readily releasable gonadotrophin builds up and becomes more and more sensitive to GnRH, so that when suddenly on the day of the LH peak much greater quantities of GnRH are secreted by the hypothalamus very large amounts of pituitary hormone are released.

What causes the change in secretion of GnRH and of the pituitary's sensitivity to it is not quite clear, but it is known that as the follicular phase progresses more oestrogen is secreted and that oestradiol produces a positive feedback effect on the pituitary and presumably also the hypothalamus-that is, under the influence of a slowly rising oestradiol level the pituitary becomes more sensitive to GnRH-and we believe also that the hypothalamus secretes more GnRH so that the stores in the pituitary are built up. Therefore early in the follicular phase there is a small response which gradually gets bigger as oestradiol levels rise until, on the day of the LH peak, there is a very big response to a large amount of GnRH.

When the response to GnRH is used as a pituitary function test in an amenorrhoeic woman there can be no normal control population with which to compare the patient's response (as there is no such thing as a 'normal' amenorrhoeic woman). Therefore the response between days 3 and 7 of the normal follicular phase is arbitrarily chosen for the reference range; it is not, however, a very good pituitary function test.
Before puberty there is only a very small LH으 response to GnRH in both males and females, but it $\overrightarrow{\vec{F}}$ increases steadily as puberty progresses. That is $\stackrel{\rho}{P}$ exactly what happens with prolonged administration of GnRH to a prepubertal patient or one who has gonadotrophin deficiency, the very small initial LH $\frac{\bar{\sigma}}{\vec{\phi}}$ response increasing until the adult picture appears. This is not so for FSH. The FSH response in both o males and females is virtually the same in a pre- $\vec{\circ}$ pubertal patient as in an adult. Thus the increasing LH response to the releasing hormones seems to be $\vec{\omega}$ the major factor in the development of puberty.

These observations have important therapeutic $?$ implications. In patients with isolated gonadotrophin is deficiency a single dose of $500 \mu \mathrm{g}$ of GnRH will. produce a fairly definite FSH response but no $\mathrm{LH}_{\dot{\infty}}$ response. A greater FSH response together with the $\circ$ secretion of LH is obtained with an infusion. When patients with either isolated gonadotrophin de- $\bar{c}$ ficiency or a hypothalamic or, surprisingly, pituitary tumour are given regular GnRH injections (for ฏ) example, $500 \mu \mathrm{g}$ of the natural form three times a day) it induces the synthesis and secretion of both $\overrightarrow{0}$ LH and FSH and, if continued long enough, induces puberty as the small initial LH response gradually increases until it exceeds the FSH response and attains the adult pattern. In men spermatogenesis appears. One patient with a craniopharyngioma, who had no gonadotrophin secretion before treat- $\frac{1}{2}$ ment, ultimately developed a sperm count of $433 \stackrel{2}{\overrightarrow{7}}$ million with $80 \%$ motility. Men may father children and women may ovulate, so there can be little doubt $\overrightarrow{ }$ that GnRH may replace gonadotrophin in treatment in most circumstances. A great advantage over gonadotrophin therapy is that if too much GnRH is? given the oestrogen levels rise so high that they begin $\frac{\text { 의 }}{3}$ to inhibit the effect of GnRH on the pituitary. This negative feedback prevents excessive gonadotrophin 0 release and hence avoids excessive stimulation of the ovary and multiple ovulation.

The releasing hormones may be used to test $D$ pituitary function. They may be used simultaneously, since the secretion of one hormone in response to one $N$ stimulus will not influence the pituitary response to another. We now perform the standard Barts triple 0 test, giving intravenous insulin, TRH, and GnRH $\omega$ simultaneously to a fasting patient after taking basal blood, for thyroxine and gonadal steroids. For three hours after the injections we collect samples for $\bar{\varnothing}$ glucose, cortisol, growth hormone, LH and FSH, TSH, and prolactin.

Sometimes-for example, in a patient with $a \stackrel{\vec{D}}{\vec{D}}$ pituitary tumour and rapidly contracting visual $\stackrel{\mathcal{Q}}{\mathcal{Q}}$ fields-the need for surgical treatment is urgent. The $\stackrel{\square}{\square}$ cortisol results must then be obtainable the same day and the $\mathrm{T} 4$ result by the following morning so that $\Omega$ 
replacement therapy can begin before proceeding to an air encephalogram, etc.

\section{GROWTH HORMONE RELEASE-INHIBITING}

HORMONE

This was discovered in 1973 (Brazeau et al., 1973). It is a cyclic compound composed of 14 amino-acids, of no clinical significance at present, but of great interest. It inhibits growth hormone release in response to every known stimulus-exercise, sleep, stress, amino-acids, hypoglycaemia, etc.-but its action is of very short duration. Since it will cut short the release of growth hormone produced by arginine infusion, and since arginine releases other hormones, it was pertinent to examine the effect of GHR-IH on these. We found that it also inhibited the release of glucagon and, even more surprisingly, of insulin. These effects are the same in patients with hypopituitarism, thus indicating that it also acts on the pancreas directly.

Further studies showed that GHR-IH also inhibits the secretion of other hormones-namely, renin (in response to frusemide-induced sodium depletion), TSH, vasoactive intestinal polypeptide (secreted by some pancreatic tumours and producing a syndrome of copious, watery diarrhoea-the Werner-Morrison syndrome), gastric inhibitory polypeptide, motilin, enteroglucagon, cholecystokynin, and secretin. It also inhibits the secretion of gastric acid (even if gastrin is given) and amylase from the salivary gland, and so on. There is a suggestion that it inhibits platelet aggregation but not everyone agrees. It does not inhibit secretion of ACTH (except in Nelson's syndrome) or prolactin.

Immunofluorescent studies have shown GHR-IH to be present not only in the hypothalamus but also in other parts of the CNS, the D-cells of the pancreas, the gastric antral area, and the upper small bowel. Thus somatostatin appears to be spread throughout the body and is believed to be part of the newly discovered 'paracrine' system. This consists of humoral agents which seem to control the secretion of adjacent cells by local diffusion rather than through the circulation.

PROLACTIN RELEASE-INHIBITING HORMONE Prolactin is not controlled by its releasing hormone, which is relatively unimportant, but by a releaseinhibiting hormone. Dopamine inhibits prolactin release and it was originally thought that dopamine released a prolactin-inhibiting factor (PIF). It has since been shown that dopamine inhibits prolactin secretion by a direct action on the anterior pituitary cells and it has recently been demonstrated in the portal capillary blood. Hypothalamic extracts release prolactin from anterior pituitary cells in vitro, but this is completely inhibited by the addition of dopamine to the medium. Dopamine is thought to be the most important PIF, though it may not be the only one. The drugs that increase prolactin secretion may be divided into dopamine receptor-blocking drugs, like metoclopramide (Maxolon) and the phenothiazines, and dopamine-depleting agents like reserpine and methyldopa. If the action of dopamine is blocked or there is no dopamine in the CNS there is no inhibition of prolactin release. Clearly, any disease which interrupts the pathways from the hypothalamus to the pituitary will cause hypersecretion or prolactin.

Bromocriptine, introduced in 1970, is a dopamine receptor agonist and inhibits prolactin secretion in this way. Its effect is of long duration, making it useful therapeutically. Males and females with high prolactin levels have hypogonadism, which recovers as soon as the prolactin levels are lowered. In females hyperprolactinaemia may cause anovular but regular cycles with a defective luteal phase or amenorrhoea; galactorrhoea may be present. In males high prolactin levels cause impotence and lactation, which disappear during treatment with bromocriptine. Treatment may need to be prolonged. One patient has been treated with bromocriptine for six years and his symptoms still recur when the drug is stopped.

\section{References}

Brazeau, P., Vale, W., Burgus, R., Ling, N., Butcher, M. Rivier, J., and Guillemin, R. (1973). Hypothalamic polypeptide that inhibits the secretion of immuno-reactive growth hormone. Science, 179, 77.

Harris, G. W. (1955). Neural Control of the Pituitary Gland. Arnold, London.

McCann, S. M., and Dhariwal, A. P. S. (1964). Hypothalamic factors which influence gonadotrophin secretion. Transactions of the New York Academy of Sciences, 27, 39.

Schally, A. V., Arimura, A., Baba, Y., Nair, R. M. G., Matsuo, H., Redding, T. W., Debeljuk, L., and White, W. F. (1971). Isolation and properties of the FSH and LHreleasing hormone. Biochemical and Biophysical Research Communications, 43, 393. 